\title{
IL SIGNORE DELLE PAROLE. LA LINGUA DEL ROMANZO IL FUOCO DI GABRIELE D'ANNUNZIO
}

\author{
Nota del m.e. MAURIZIO VITALE (*)
}

(Adunanza del 28 giugno 2018)

SunTO. - La mia nota consiste nell'esame approfondito delle forme linguistiche e stilistiche del romanzo "Il Fuoco". Lo scrutinio di tali forme attesta la presenza, nella lingua di straordinaria ricchezza e varietà e nello stile ricco di figure retoriche, di modi anticheggianti e culti che connotano l'alta letterarietà e la magnificenza espressiva di D’Annunzio signore delle parole.

ABSTRACT. - The following article deeply examines the linguistic and stylistic forms of the novel "Il Fuoco". The analysis of such forms bears witness to the presence, in a language unusually rich and varied besides a style full of figures of speech, of polished and old-fashioned expressions, which characterize the high literary tone and the expressive grandeur of D'Annunzio "king of the words".

Dell'opera di D'Annunzio romanziere (autore del Piacere, dell'Innocente, del Trionfo della morte, di Giovanni Episcopo, delle Vergini delle rocce e, quindi del Fuoco), pur esistendo, nelle brillanti analisi da parte di illustri critici, fini e sottili considerazioni, specialmente di stile e di lessico, manca tuttavia un approfondito ed esteso esame delle forme retoriche e linguistiche che connotano l'alta letterarietà di D'Annunzio artefice della parola. La mia nota mira alla definizione, per la prosa di un romanzo, della forma espressiva dannunziana della narrazione nei suoi tratti qualificanti di stile e di lingua.

(*) Università degli Studi di Milano, Italy. E-mail: info@istitutolombardo.it 
Riassumerò il contento del romanzo, perché meglio e più chiaramente si possano intendere i tratti formali con i quali è espresso. Il Fuoco, scritto tra il 1896 e 1899 e pubblicato nel 1900, nella sua prosa alta e dotta, è il romanzo in sostanza autobiografico della intensa passione tra il giovane già noto poeta e drammaturgo Stelio Ėffrena (Gabriele D'Annunzio) e la matura grande e celebre attrice teatrale FoscarinaPèrdita (Eleonora Duse), spesso turbata dalla gelosia della Foscarina per la giovane amica dal canto soave Donatella Arvale, alla quale talora Stelio, suo ammiratore, tacitamente invaghito, rivolge il pensiero, La narrazione prende avvio, dopo la passeggiata in gondole di Stelio e della Foscarina evocante la morte dell'Estate, dalla lunga e calda allocuzione di Stelio Èfffrena, l'«Imaginifico» (nella quale si fa cenno dell'incontro del poeta con i coloristi veneti Tintoretto, Veronese, Giorgione) tenuta in lode di Venezia, città magnifica «d'acqua e di pietra», con grande successo davanti a una folle immensa e in presenza della Regina d'Italia, nella sala del Gran Consilio del Palazzo Ducale. E la narrazione séguita con l'esposizione, ricca di animata vivezza, di una varia serie di eventi nei quali si svolge il tormentato rapporto amoroso tra Stelio e la Foscarina nel quadro della fervida vita veneziana, fatta anche di discussioni sull'arte e sulla musica (su Benedetto Marcello, sul «divino» Monteverdi e quindi sull'opera di Wagner). Nel corso del romanzo Stelio, già evocatore dei classici greci, in particolare di Eschilo e Sofocle, e dei miti classici, descrive all'amico Glauro (il filosofo estetizzante Angelo Conti) il soggetto del dramma che sta componendo, La Città morta (scritta nel 1896 e rappresenta a Parigi nel 1868) e ne spiega il profondo significato. Il rapporto, acceso e turbolento, tra Stelio e la Foscarina sembra interrompersi, in un distacco amichevole, con la partenza della Foscarina per l'America.

D'Annunzio, scrittore di larga dottrina e di lungo studio letterario, di spirito insieme classico e moderno, palesa nel Fuoco (che l'autore considerava superiore ad ogni altro suo libro) di possedere la «scienza della parole» e mostra di avere penetrato la lingua della tradizione per ricavarne «i tesori lentamente accumulati di secolo in secolo» e di avere già fruttuosamente meditato su strumenti lessicografici moderni e su opere specialistiche.

Nella convinzione di riunire nel suo stile «le più diverse virtù della parola scritta», nel Fuoco ha adottato una lingua orientata verso l'antico, impiegando singolarità espressive, grande varietà e ricchezza di voci e di forme, compresenza di tratti eletti e correnti per le medesime voci, singolari ritmi espressivi. 
Rispondono al gusto della rarità singolare le voci eleomele sost. "balsamo oleoso e dolce", esegete sost. "interprete, critico", musurgo sost. "suonatore di musica", multanime agg., versicolore agg." di vario colore".

Attestano la varietà e la ricchezza della lingua:

i latinismi: grafici conscienza, construtto, conspetto; etimologici academia, comento, drama: lessicali animula, cucurbita, glandule, vagula;

i grecismi: anadiomene "che sorge dal mare", criselefantina agg. "fatta d'avorio e di oro", lampadeforie agg. plur. "portatrici di fiaccole", perittero "edificio che ha intorno colonne"

gli arcaismi volgari: adunazione, chiragra "gotta", magione, plenitudine, ploro "lamento"

le voci e le forme letterarie: amaritudine, aulentissimo, conquidere "conquistare", cubito "gomito", doglia, imo, lene, letifera "che reca morte", maritaggio, nimistà, onusta "carica", opimo, pampinifero adorno di pampini", procella "tempesta", recidere, rilucere "risplendere", salutazione salito", vespero, vipistrello "pipistrello"

le forme letterarie fonetiche, morfologiche e sintattiche: nel vocalismo novo, foco, laude, divoto, romore, simiglianza, eguale, escire, gitta $3^{\text {a }}$ sing., bevere; nel consonantismo ripa, belli occhi, benefizio, palagio, acciaro, armarii plur. "armadi"; nella morfologia i pronomi elle, elleno, cui complemento oggetto, i nomi il prigioniere, l'avventuriere, la carcere, e i plurali annunzii, artifiziz; le forme verbali veggo, io assisteva, offerse $3^{a}$ sing., i participi istrutto, construtto; la preposizione pel per il”, gli avverbi anco, omai; nella sintassi il participio presente con valore verbale ( $i$ simulacri dei numi... spiranti divinita) ), l'accusativo con l'infinito di tradizione classicheggiante (a quel retaggio che il messaggero le annunciava essere ancóra intatto e recuperabile);

attestano altresì la ricchezza lessicale:

i termini tecnici dell'arte marinara bompresso, calcagnolo, forcola, scala penzola (con i nomi di alcune imbarcazioni veneziane bucintoro, bissona, bragozzo, burchio, peata, peota); e i termini dell'arte vetraria anzipetti "parapetti", canna di fero, coppe, forno fusorio;

i dialettismi veneziani calandrine "allodole", squero "cantiere per riparo gondole", velme "melme", vissole "ciliegie";

gli esotismi: gli ispanismi galgo "veltro", rovana (stoffa) "di colore rossiccio", l'arabismo sloughi levriero nordafricano"; 
i neologismi dannunziani aromàle agg. "che ha la fragranza di aroma", esegete "critico, interprete", pentafora "finestra di cinque luci eguali", sinfoneti plur. "compositori di sinfonie";

e infine:

la terminologia degli strumenti musicali arpicordio, cembalo, clavicordio, eptacordo, liuti;

il lessico ampio dei colori di gusto impressionistico azzurro, bianco - biancastro-biancheggiare, cerulo, cinereo, croceo, fulvo, giallo - giallastro, nero - nerastro- nereggiare - nerebianche plur., opalino, paonazzo, rosso - rossastro - rosseggiare, verde - verdastro - verdazzurre plur. - verdegiallo;

il lessico insistito dei sentimenti gravi e dell'indeterminatezza ricorrente in toni alti angoscia, atroce, orrore, orribile, terrore, terribile, immenso, infinito

gli elativi di uso imponente, nella grandigia espressiva: oltre a oltrapiacente e oltrepossente, tra gli altri, acutissima vigilanza, altissimi zoccoli, altissimo silenzio, vita antichissima, occhi attentissimi, vin dolce e aulentissimo, bellissimo avventuriere, monache...delicatissime, altissime forme, fierissimo Malatesta, lievissima reclinazione, cieli lontanissimi, lunghissimo cammino, nobilissimo animo, novissimo senso musicale, volto pallidissimo;

mostrano la pluralità, l'opulenza linguistica i tratti culti e correnti impiegati per le stesse voci e le stesse forme: romore / rumore; escire/ uscire; gittare/ gettare; edifizio/ edificio; palagio/ palazzo; acciaro / acciaio; armariil armadi; gridal gridi; veggo/ vedo; andavo / io assisteva; apersel apri; scopersel scopri;

concretano il ritmo melodico della prosa talune cadenze gradite ripetutissime nei suffissi o di lieve sonorità: balenio, cinguettio, crepitio, gridio, luccichio, mugolio, romorio, sciacquio, sfolgorio, tremolio, turbinio; o soprattutto di sonorità altisonante con voci proparossitone e in negativo iniziale: inaccessibile, incredibile, inestinguibile, inflessibile, insostenibile, intangibile, irresistibile, ma specialmente inafferrabile, incalcolabile, inconsolabile, indomabile, inesplorabile, inestricabile, inuppugnabile, inviolabile, impenetrabile, irreparabile.

Egli ha infine sottoposto la sua prosa, già per sua natura dotta, a una lezione retorica raffinata e sottile con l'impiego di figure retoriche di espressione: 
la perifrasi: per contadino nomo della gleba; per marinaio uomo destinato all'alto mare

la metafora: odor febrile, malinconie cineree, sonoro silenzio, mollezza opalina

la sinestesia:, tepore biondo, silenzio immobile, sordità del silenzio_ di parola:

l'accumulazione verbale con sequenze di aggettivi, nomi e verbi: vi veggo pallido e pensieroso; un'anima ingenua e magnifica; l'acqua era umida molle e cinerina; La forza e la fiamma sono in voi; per vedermi fiorire e fruttificare; o con sequenze di membri o cola, il cui dispiegamento lineare determina così una sintassi per lo più paratattica, in una amplificazione magniloquente: Ella cosi blandiva l'amico delicatamente, lo avvolgeva in una continua lusinga, lo esaltava in una continua lode; Poi, d'un tratto, risaliva nell'aria lucida il clamore, si frangeva su per la snella foresta marmorea, superava le fronti delle statue, attingeva i pinnacoli e le croci, si disperdeva nella lontananza crepuscolare; ecc. ecc.

la ripetizione di interi passi all'interno della narrazione che sembra corrispondere a una ripresa di motivi in una partitura musicale e che disvela quindi una sorte di ritmo musicale continuato : p. 571 per sorridere attonita come una fanciulla cui si mostri un libro figurato - p. 830 per sorridere attonita come una fanciulla cui si mostri un libro figurato; p. 649 Scintillavano gli astri, ondeggiavano gli alberi dietro il capo di Perdita, si profondava un giardino - 664 Scintillavano gli astri, ondeggiavano gli alberi dietro il capo di Perdita, si profondava un giardino

l'anastrofe aggettivale che accentua la tendenza alla inversione: approdante all'antica riva; l'imagine sua propria nel poetico specchio; nelle ancone degli interni altari

la tmesi o distanziazione dell'aggettivo dal nome: e di più aggiungevasi al sentimento ch'egli aveva della sua persona consueto; rimembrava...il loro garrito, già onta delle flotte fuggiasche, implacabile

l'anafora: Egli la vedeva integra e singolare...Egli la vedeva; l'immenso clamore saliva saliva per l'adunazione dei marmi; Un sorriso infinito vi si diffuse, infinito; e il polittoto: ella ruppe in singhiozzi; Singhiozzò singhiozzò, là, sopra il petto di lui

l'allitterazione, per lo più in omeoteleuti: al sentimento che si prova dopo aver offeso e leso, senza volere, un infermo; straziante inebriante contrasto; un atto di purificazione e di liberazione 
e, infine, figure di pensiero:

l'antitesi: anelante... a conoscere qualche libera ebrezza di gioia e di dolore; il soffio delle parole sembrava alterare le luci e le ombre; concentrazioni e dispersioni rapidissime di pensieri si succedevano

l'ossimoro: manifestarono l'uno all'altra un grazioso orrore; sembrava quasi mosso da un dispregio allegro

il chiasmo: per trovarvi «invenzioni nobilissime» $e$ «infinite cose»; uno spirito di vita, fatto d'aspettazioni appassionate e da contenuto ardore; tempo... di stanchezza smaniose, di chiuso orrore

la comparazione intensissima, come amplificazione lineare dell'espressione: che ingemmano la casa dei Dario come una cortigiana decrepita; Ella giace nella bara funebre, vestita d'oro come una dogaressa, come una Loredana; $i$ grandi alberi trascolorati fiammeggiarono sul loro capo come cortinaggi di porpora che s'incendiassero

da ultimo le figure grammaticali:

accusativo di relazione, di tradizione classicheggiante: Era forse il divino pianto della Minoide protesa invano le braccia deluse; riappariva umile e pallida sotto la veste della messaggera, curva il capo, spenta lo sguardo figura etimologica: si abbandonerà domani all'incanto della mistica per sognare un sogno di voluttà infinito; Per quel velo guadò la laguna che tremolò di quel tremolio

Ha dunque l'artefice attuato nel romanzo una grammatica (grafia fonetica, morfologia, sintassi) e un patrimonio lessicale ispirati a un ideale di lingua di singolare e personale nobiltà con l'intento di concretare la magnificenza della elocuzione, assumendo per essa valori linguistici non comuni, talora straordinari.

E ha altresì l'artefice adunato con sapiente industria, come si rileva dalla lezione retorica, un imponente materiale espressivo (parafrasi, antonomasia, copia verborum, copia locutionum), un accorta inversione dell'ordine sintattico (prolessi, tmesi, chiasmo), mostrando altresì un gusto elegante per la contrapposizione concettuale (antitesi, ossimoro), una inclinazione per le sonorità inusuali (allitterazione), una viva tendenza all'accentuazione ripetitiva (anafora, ripetizioni), una singolare sensibilità per la sinestesia e un gradimento grandissimo per la similitudine o comparazione e la ripetizione degli stessi passi per ritmo melodico, 
La scrittura del romanzo, scrutinata nelle sue diverse parti di lingua e di stile, nei suoi molteplici registri, nella diversità delle componenti del suo lessico, nella costanza dei suoi toni alti, nella prevalenza dei modi culti e letterari, attesta, dunque, per varietà e ricchezza, nell'autore, un singolare ed eccezionale Signore della scienza delle parole. 
